\title{
Magnetic properties of densely packed arrays of Ni nanowires as a function of their diameter and lattice parameter
}

\author{
M. Vázquez, ${ }^{\text {a) }}$ K. Pirota, M. Hernández-Vélez, V. M. Prida, D. Navas, R. Sanz, \\ and F. Batallán \\ Instituto de Ciencia de Materiales de Madrid, CSIC 28049 Madrid, Spain \\ J. Velázquez \\ CAI-XRD, Fac. C. Químicas, Univ. Complutense, 28040 Madrid, Spain
}

(Presented on 6 January 2004)

\begin{abstract}
High-quality densely packed hexagonal arrays of $\mathrm{Ni}$ nanowires have been prepared by filling self-ordered nanopores in alumina membranes. Nanowires with different diameter $d(18-83 \mathrm{~nm})$ and lattice parameter $D(65$ and $105 \mathrm{~nm})$ have been studied by atomic force, high resolution scanning electron microscopies, Rutherford backscattering, and vibrating sample magnetometer techniques. Axial loops coercivity and remanence decrease with increasing ratio diameter to lattice parameter, $r$, until nanowires start to interconnect locally. Additionally, hysteresis of in-plane loops increases with packing factor. In order to interpret the experimental results, multipolar magnetostatic interactions among nanowires with increasing ratio $r$ are considered. (C) 2004 American Institute of Physics. [DOI: 10.1063/1.1687539]
\end{abstract}

\section{INTRODUCTION: PREPARATION AND STRUCTURE CHARACTERIZATION OF Ni NANOWIRE ARRAYS}

Studies on highly ordered arrays of magnetic nanowires are recently attracting a growing interest. This ordering, together with the intrinsic nature of the nanomagnets, gives rise to outstanding cooperative properties different from bulk and even from film systems. ${ }^{1,2}$ Arrays of magnetic nanowires can be produced, among other techniques, ${ }^{3}$ using nanoporous alumina membranes as templates. Highly ordered hexagonal symmetry is induced by the self-ordering process of nanopore formation. Arrays of nanowires typically $20-200 \mathrm{~nm}$ in diameter, around $1000 \mathrm{~nm}$ long and separated 30-200 nm are very suitable systems for micromagnetic studies and for potential technological applications.

Studies on general magnetic behavior have been reported on arrays of nanowires of $\mathrm{Fe}, \mathrm{Ni}, \mathrm{Co}$, and their alloys. ${ }^{4-6}$ Individual $\mathrm{Ni}$ and $\mathrm{Fe}$ nanowires are taken in a first approximation to be single-domain with longitudinal magnetization mainly due to shape anisotropy. Magnetization reversal by curling rotational model combined with nucleationpropagation processes for nanowires with diameter in the range of around $50-400 \mathrm{~nm}$ has been proposed for individual nearly noninteracting nanowires. In the case when the wire diameter $d$ is smaller than the exchange correlation length (for $\mathrm{Fe}, \mathrm{Co}$, and $\mathrm{Ni}$ it lies in the order of $10-50 \mathrm{~nm}$ ), magnetization should reverse at unison by coherent rotation. Nevertheless, some discrepancies have been found regarding those expectations. The determination of actual closure structures at the ends and of the nonaxial components of magnetization seem to be rather important to acquire deeper knowledge of the reversal process. Finally, magnetostatic interactions between nanowires should play a determinant

a)Electronic mail: mvazquez@icmm.csic.es role, specially in the case of densely packed arrays.

A first objective of this work has been the controlled production of densely packed highly ordered nanowire arrays. As discussed previously, ${ }^{7,8}$ a deep understanding of how strongly nanowires are magnetically coupled is quite important since it would determine, for example, the size of magnetic bits of information for magnetic storage. Here, we study in further detail the importance of magnetostatic interactions in the arrays, introducing the influence on the magnetic behavior of geometrical characteristics particularly for the case when nanowire diameter $d$ reaches values comparable to interwire distance $D$.

Nanoporous alumina membranes with hexagonal ordering have been prepared by a two-step anodization process. ${ }^{6,9}$ Nanopores form by self-assembling process, and parameters of first anodization determine the ordering degree of final pore arrays and the interpore distance. The time of the second anodization process determines the length of nanopores, and their diameter can be finer controlled by subsequent treatment in phosphoric acid. For the present work, diameter of nanowires, as determined by AFM and HRSEM techniques, ranges between 18 and $33 \mathrm{~nm}$ for interwire distance of $65 \mathrm{~nm}$ employing sulphuric acid as electrolytic bath, and between 35 and $83 \mathrm{~nm}$ diameter for interwire distance of 105 $\mathrm{nm}$ using oxalic acid as bath. Treatments in phosphoric acid to increase the pore diameter in both series of membranes lasted until 30 and $20 \mathrm{~min}$, respectively. For longer treatments, the arrays deteriorate as nanopores start to interconnect locally.

Nanopores are afterwards filled with Ni by electrodeposition with alternating pulse voltage technique. Figure 1 shows a HRSEM image showing a surface polydomain structure. Long range order of nanowires is deduced from the fast Fourier transform (see inset) which has been taken from a very large area of the real image (about $24 \mu \mathrm{m}^{2}$ ). The 


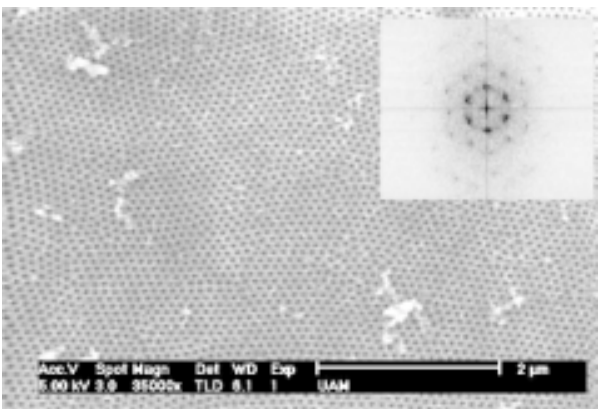

FIG. 1. Real surface HRSEM image of a nanoporous alumina membrane. The inset shows the FFT calculated of a large $\left(24 \mu \mathrm{m}^{2}\right)$ area of the nanoporous membrane image.

control of nanopore filling is performed by Rutherford backscattering technique, RBS. ${ }^{10}$ Figure 2(a) shows the RBS experimental results. The observed sharp peak denotes a $\mathrm{Ni}$ excess on the surface of the sample (which is later removed by careful polishing). The Ni filling of the pores is observed just to the left side of that peak. Figure 2(b) shows the atomic composition profile analyzed from the surface to the bottom of the sample. This figure confirms the Ni overflow on the membrane surface, as well as a continuously increasing $\mathrm{Ni}$ content when approaching the bottom. In a first approach we consider that the increasing yield to lower energies [up to channel 782, see Fig. 2(a)] could be due to a partial lack of fully filling of some nanopores. A complete interpretation of this kind of spectra is introduced elsewhere..$^{10}$ The final optimization of the geometrical quality of the array includes a polishing of the membrane surface to remove the Ni overflowing and an ion-beam etching of its bottom after aluminum substrate removing, to eliminate the dendrites accumulated during the pores formation.

\section{MAGNETIC BEHAVIOR AND ITS ANALYSIS}

Magnetic characteristics of the nanowire arrays have been measured by VSM magnetometer at room temperature. From the longitudinal and in-plane hysteresis loops magnetic parameters such as remanence, coercivity, or susceptibility are evaluated. The existence of an effective longitudinal magnetic anisotropy (parallel to the nanowire axis) can be checked by comparing longitudinal and transverse (in-plane of the membrane) hysteresis loops. For example, from the loops for an hexagonal array of Ni nanowires $35 \mathrm{~nm}$ in diameter and $105 \mathrm{~nm}$ in lattice parameter, an effective axial
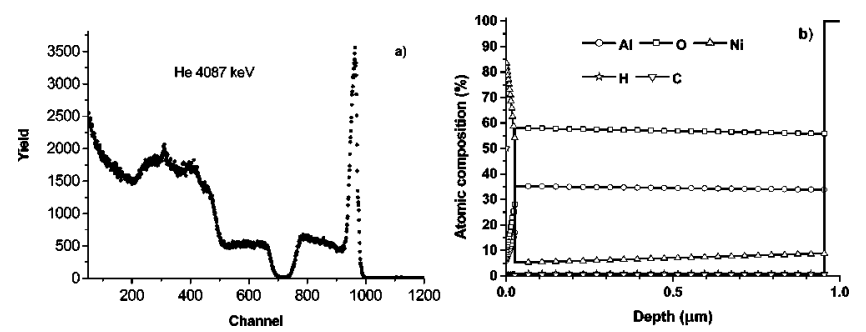

FIG. 2. (a) Experimental (4087 keV radiation) RBS spectrum in a membrane with Ni overflow on its surface. (b) Profile of atomic composition in $\%$ calculated from the spectrum in (a).
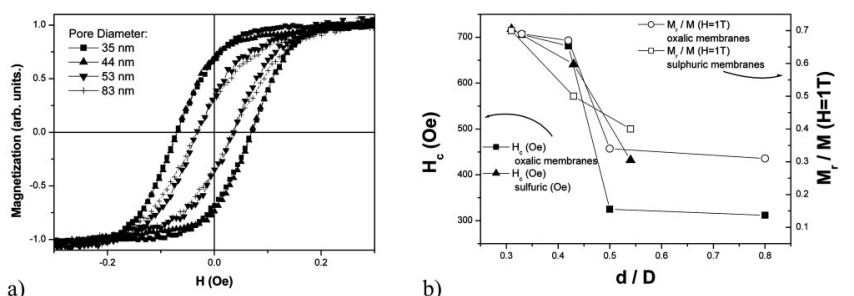

FIG. 3. (a) Longitudinal hysteresis loops as a function of the nanowire diameter for Ni nanowires separated $105 \mathrm{~nm}$ having diameters in the range from 35 to $83 \mathrm{~nm}$. (b) Dependence of coercivity (filled symbols) and remanence (unfilled symbols) as a function of the ratio $d / D$ for the series of membranes prepared in oxalic and sulphuric acids.

magnetic anisotropy is deduced with anisotropy field of around $2.5 \mathrm{kOe}$. That allows us to consider in a first approximation individual nanowires in such array to be nearly single domains.

Figure 3(a) shows the longitudinal hysteresis loops for nanowires ranging in diameter between 35 and $83 \mathrm{~nm}$ in an array with lattice parameter of $105 \mathrm{~nm}$. The dependence of coercivity and remanence of the loops on the ratio of nanowire diameter to lattice parameter, $r=d / D$, is shown in Fig. 3(b). As it can be observed, both magnitudes decrease with increasing ratio $r$. A similar behavior is observed for the series of nanowires with $65 \mathrm{~nm}$ lattice parameter ranging in diameter between 18 and $33 \mathrm{~nm}$.

In order to interpret this behavior, let us analyze the influence of the increasing diameter of the nanowires. As the diameter increases, nanowires effectively approach each other since the interwire distance is kept constant, and consequently they start to get magnetostatically coupled. ${ }^{8,11}$ Magnetostatic interactions favor an antiparallel distribution of magnetization in neighboring nanowires. For nanowires initially magnetized in the same direction, the increase of magnetostatic interaction results in the magnetization reversal of $N$ nanowires. Assuming that the reversal of an individual nanowire produces a decrease of magnetostatic energy $E_{v}$ that equals the magnetic anisotropy barrier $\Delta E$, the macroscopic coercivity will be: ${ }^{11}$

$$
H_{C}=\frac{2 K}{\mu_{0} M_{S}}\left[1-\left(\frac{N\left|E_{v}\right|}{K}\right)^{1 / 2}\right],
$$

where $2 K / \mu_{0} M_{s}$ denotes the intrinsic coercivity due to the magnetoelastic or magnetocrystalline anisotropies, $K$, and $E_{v}$ the magnetostatic interaction between two nanowires. Such interaction has been derived considering each nanowire to be homogeneously magnetized, due to their uniaxial magnetic anisotropy, with magnetic charges at both ends, $\sigma$ $=/ M /$. This interaction energy, including multipolar components, has an expression $E_{v}=\left(\mu_{0} / 8 \pi\right) M_{1} M_{2}(D / L) r^{2}[1$ $\left.-\left[1+(L / D)^{2}\right]^{-1 / 2}\right]$ (valid for $d / L \ll 1, L$ being the length of the nanowires and $M_{i}$ the axial component of magnetization in each nanowire. ${ }^{8,12}$ ) Thus, according to Eq. (1), a linear reduction of coercivity with the parameter $r$ is expected. Nevertheless, as observed in Fig. 3(b), the evolution of coercivity loses linearity for the larger value of $r$ seemingly as a consequence of a modification of the reversal mode due to an effective change of the magnetization easy axis from axial 


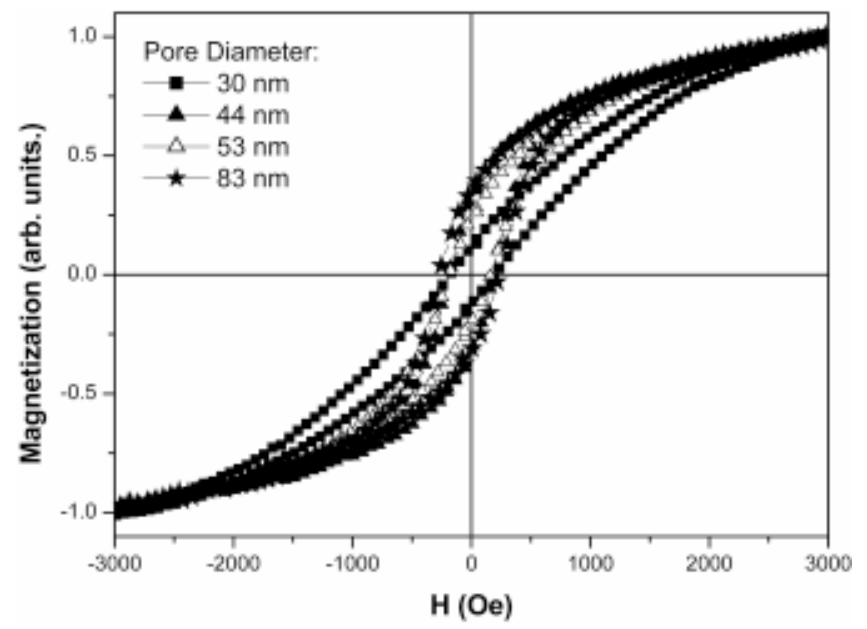

FIG. 4. Transverse hysteresis loops as a function of the nanowire diameter for $\mathrm{Ni}$ nanowires separated $105 \mathrm{~nm}$ having diameters in the range from 35 to $83 \mathrm{~nm}$.

to in plane of the membrane. Although derived in a different way, ${ }^{13}$ a similar behavior is expected considering the dependence of the effective shape anisotropy with the packing density, $H_{\mathrm{Sh}}=M_{s}\left[1-\left(\pi / 3^{1 / 2}\right) r^{2}\right]$.

Additional information is supplied from in-plane hysteresis measured for all the nanowire arrays of the series mentioned above. The shape of the loops shown in Fig. 4 evolves with the ratio $r$ indicating that the magnetization easy axis gradually rotates towards an in-plane direction. Particularly, coercivity remains almost constant (around $180 \mathrm{Oe}$ ) with the increasing diameter but for the most dense array (260 Oe).

It should be mentioned that a final increase of longitudinal coercivity and remanence has been observed for the case when long treatment in phosphoric acid makes nanopores to locally interconnect giving rise finally to collapse into a continuous film. For low density of nanowires, individual nanowires can be approached to be single-domain structured due to shape anisotropy. In this case, the magnetostatic interaction, being relatively weak, simply determines a reduction in remanence and coercivity. As the ratio $r=d / D$ becomes large enough, the effective easy axis rotates from axial to transverse direction in the plane of the membrane. In this case, the nearly single domain structure of nanowires con- verts to multidomain and finally the magnetization process is modified. Similar effects have been analyzed in cylindrical dots. $^{11}$

\section{CONCLUSIONS}

Densely packed arrays of Ni nanowires embedded in porous alumina membranes have been prepared and structurally characterized. Their high ordering degree with hexagonal symmetry of nanopores and their filling have been carefully studied. We report on the magnetic behavior of arrays of $\mathrm{Ni}$ nanowires for various diameter: and lattice parameter of the hexagonal symmetry array. It is experimentally found that both coercivity and remanence decrease with diameter to lattice parameter ratio. Results are discussed considering the increasing multipolar magnetostatic interactions within the array as the ratio $r$ increases.

\section{ACKNOWLEDGMENTS}

The authors wish to thank Dr. K. Nielsch, Dr. F. Paszti, and Dr. A. Climent for analysis of results. The work was supported by the Autonomous Community of Madrid under Project No. CAM2002-0405.

${ }^{1}$ D. Appell, Nature (London) 419, 553 (2002).

${ }^{2}$ See, J. Magn. Magn. Mater. 249 (2002).

${ }^{3}$ J. I. Martín, J. Nogués, K. Liu, J. L. Vicent, and I. K. Schuller, J. Magn. Magn. Mater. 256, 449 (2003).

${ }^{4}$ D. J. Sellmyer, M. Zheng, and R. Skomski, J. Phys.: Condens. Matter 13, R433 (2001).

${ }^{5}$ P. M. Paulus, F. Luis, M. Kröll, G. Schmid, and L. J. de Jongh, J. Magn. Magn. Mater. 224, 180 (2001).

${ }^{6}$ K. Nielsch, R. B. Wehrspohn, J. Barthel, J. Kirschner, S. F. Fischer, H. Kronmüller, T. Schweinböck, D. Weiss, and U. Gösele, J. Magn. Magn. Mater. 249, 234 (2002).

${ }^{7}$ M. Vázquez, K. Nielsch, P. Vargas, J. Velázquez, D. Navas, K. R. Pirota, M. Hernández-Vélez, E. Vogel, J. Cartes, R. B. Wehrspohn, and U. Gösele, Physica B 343, 395 (2004).

${ }^{8}$ J. Velázquez, K. R. Pirota, and M. Vázquez, IEEE Trans. Magn. 39, 3049 (2003).

${ }^{9}$ K. R. Pirota, D. Navas, M. Hernández-Vélez, K. Nielsch, and M. Vázquez, J. Alloys Compd. (to be published).

${ }^{10}$ M. Vázquez et al., Invited at SMM'03 Conf., Düsseldorf, 2003, M. Hernández-Vélez et al. (unpublished).

${ }^{11}$ K. Yu. Guslienko, Phys. Lett. A 278, 293 (2001); W. Scholz, K. Y. Guslienko, V. Novosad, D. Suess, T. Schrefl, R. W. Chantrell, and J. Fidler, J. Magn. Magn. Mater. 266, 155 (2003).

${ }^{12}$ J. Velázquez, D. Laroze, P. Vargas, and M. Vázquez (unpublished).

${ }^{13}$ A. Encinas-Oropesa, M. Demand, L. Piraux, I. Huynen, and U. Ebels, Phys. Rev. B 63, 104415 (2001). 